\title{
Mapping non-host resistance to the stem rust pathogen in an interspecific barberry hybrid
}

\author{
Radhika Bartaula', Arthur T. O. Melo ${ }^{2}$, Sarah Kingan ${ }^{3}$, Yue $\mathrm{Jin}^{4}$ and lago Hale ${ }^{2^{*}}$ (D)
}

\begin{abstract}
Background: Non-host resistance (NHR) presents a compelling long-term plant protection strategy for global food security, yet the genetic basis of NHR remains poorly understood. For many diseases, including stem rust of wheat [causal organism Puccinia graminis (Pg)], NHR is largely unexplored due to the inherent challenge of developing a genetically tractable system within which the resistance segregates. The present study turns to the pathogen's alternate host, barberry (Berberis spp.), to overcome this challenge.

Results: In this study, an interspecific mapping population derived from a cross between Pg-resistant Berberis thunbergii (Bt) and Pg-susceptible B. vulgaris was developed to investigate the Pg-NHR exhibited by Bt. To facilitate QTL analysis and subsequent trait dissection, the first genetic linkage maps for the two parental species were constructed and a chromosome-scale reference genome for Bt was assembled (PacBio + Hi-C). QTL analysis resulted in the identification of a single $13 \mathrm{cM}$ region ( 5.1 Mbp spanning 13 physical contigs) on the short arm of Bt chromosome 3. Differential gene expression analysis, combined with sequence variation analysis between the two parental species, led to the prioritization of several candidate genes within the QTL region, some of which belong to gene families previously implicated in disease resistance.

Conclusions: Foundational genetic and genomic resources developed for Berberis spp. enabled the identification and annotation of a QTL associated with Pg-NHR. Although subsequent validation and fine mapping studies are needed, this study demonstrates the feasibility of and lays the groundwork for dissecting Pg-NHR in the alternate host of one of agriculture's most devastating pathogens.
\end{abstract}

Keywords: Wheat, Stem rust, Barberry, Non-host resistance, Durable resistance, Reference genome

\section{Background}

Stem rust, caused by the fungal pathogen Puccinia graminis $(P g)$, has for millennia been one of the most destructive diseases of wheat and related small grains [1-3]. Effective control of the disease was realized in the middle of the twentieth century through the concerted development of resistant wheat varieties and the removal of $P g$ 's alternate host, common barberry (Berberis vulgaris L.), from major wheat growing areas $[3,4]$. In the last 20 years, however, the emergence of new virulent stem rust races has rendered some long-used resistance genes ineffective [5, 6]. For example, when the wheat stem rust race $U g 99$ was first

\footnotetext{
*Correspondence: iago.hale@unh.edu

${ }^{2}$ Department of Agriculture, Nutrition, and Food Systems, University of New Hampshire, Durham, NH 03824, USA

Full list of author information is available at the end of the article
}

detected in East Africa in 1998, more than $80 \%$ of the world's wheat germplasm was estimated to be vulnerable to its unprecedented virulence on the widely-deployed resistance gene $S r 31$ [7]. The rapid distribution and continued evolution of the $U g 99$ family of races, combined with recent stem rust outbreaks in Europe [8], underscore the need for new sources of resistance [9]. Traditionally, such new sources have been sought almost entirely from within the diverse Triticum genepool. Although translatability to wheat improvement may be less straightforward, or potentially even unachievable, a complementary approach may to look beyond this genepool for potential mechanisms of non-host resistance (NHR) to the complex $P g$ pathogen.

NHR is a form of resistance in which all individuals of a potential host species exhibit immunity to all individuals (e.g. races) of a potential pathogen [10]. As the

(c) The Author(s). 2019 Open Access This article is distributed under the terms of the Creative Commons Attribution 4.0 International License (http://creativecommons.org/licenses/by/4.0/), which permits unrestricted use, distribution, and 
most common form of disease resistance and one that possesses intrinsic durability, NHR presents a compelling strategy for achieving broad-spectrum, durable protection against many plant pathogens, including the causal organism of wheat stem rust [11, 12]. The genetic mechanisms underlying $P g$-NHR remain largely unknown, especially in comparison to the relatively well-studied mechanisms of race specific and quantitative, race nonspecific host resistance. Over the past decade, however, efforts have mounted to understand NHR to rust pathogens using various model and non-model plants. Many plant species, including Arabidopsis thaliana, Brachypodium distachyon, rice, barley, and cowpea [13-18], have been used to study NHR to P. striiformis f. sp. tritici, the causal organism of wheat stripe rust. In contrast, NHR to the wheat stem rust pathogen $P g$ has thus far been studied only in rice [13], as distinct from the studies of intermediate $P g$ resistance conducted in barley and B. distachyon $[19,20]$.

As the only globally important small grain immune to all known rust diseases, rice (Oryza spp.) presents a logical potential source of $P g$-NHR genes. Genetic studies of $P g$-NHR in rice are difficult, however, precisely because populations of non-hosts fail, by definition, to segregate for resistance. Although some limited progression of $P g$ infection has been shown in rice, thus raising the possibility of dissecting $P g$-NHR in that system, the infection process exhibits little variation, requires tedious microscopic studies to characterize, and ultimately fails to complete [13]. As an alternative to rice, the Berberis- $P g$ system was recently proposed as a tractable pathosystem for studying the genetics of $P g$-NHR [21]. Numerous species within the highly diverse Berberis, or barberry, genus are susceptible to $P g$ infection (e.g. European barberry $B$. vulgaris L., the target of massive eradication efforts from wheat-growing regions in the twentieth century) $[22,23]$. Others, however, are considered non-hosts. Japanese barberry B. thunbergii DC., for example, is considered a non-host of $P g$ due to two lines of evidence: 1) Over nearly a century of extensive testing at the USDA Cereal Disease Lab, no $P g$ infection has ever been observed in the species [24-33], and 2) No $P g$ infection has been observed on $B$. thunbergii under natural conditions, despite rampant proliferation of the species in the landscape. Because hybridization between such host and non-host species are known to occur in nature (e.g. B. xottawensis C.K. Scheid) [34], populations of interspecific barberry hybrids present a potential means of mapping and dissecting the genetic basis of $P g$-NHR.

The barberries are a compelling model for other reasons as well. Unlike rice, which has no known co-evolutionary relationship with $P g$, barberries are thought to be one of the first eudicots parasitized by the rusts (Fig. 1). Indeed, multiple lines of evidence support the idea that the barberries may have played an important role in the evolution of the rust fungi. First, Berberis spp. host a wide diversity of rusts, including numerous macrocyclic, heteroecious species of Puccinia (e.g. Pg, P. striiformis, P. montanensis, $P$. brachypodii, $P$. pigmea, $P$. koeleriae, and $P$. arrhenatheri), a number of autoecious rusts (e.g. Cumminsiella spp., belonging to Pucciniaceae; Edythea spp., belonging to Uropyxidaceae; and Pucciniosira spp., belonging to Pucciniosiraceae), and even some anamorphic rusts (e.g. Acedidium and Uredo spp.). Second, only slight morphological differences exist among the teliospores of the various macrocyclic rusts [35], suggesting a single evolutionary origin of these pathogens. Third, a recent palaeobotanical finding of $B$. wuyunensis from a sediment layer between 55 to 65 million years ago in northeastern China suggests that the barberries are one of the earliest groups of angiosperms [36].

More specific to the grass rusts, there are eight known Puccinia spp. that complete their sexual (aecial) stage on barberry and their asexual (uredinial and telial) stages on graminaceous plants from the Poaceae family. This relationship, in combination with the relative ages of these two plant families, suggests that Puccinia spp. likely parasitized the Berberidaceae prior to their host expansion to the grasses. Today, the genus Puccinia is comprised of more than 2000 species; and within that diverse genus, host jump rather than co-speciation is believed to be the primary means of speciation [37]. As more recent examples, a host jump from Poaceae to Ranunculaceae likely produced the $P$. recondita complex and its aligned species, a jump to Liliaceae likely produced $P$. hordei and its aligned species, and a jump to Oxalidaceae likely produced $P$. sorghi and its aligned species. Because the relationship between barberries and the rusts likely predates such speciation (Fig. 1), it is of fundamental interest to probe the mechanism(s) of NHR exhibited by some contemporary species of barberry.

In this study, an interspecific B. xottawensis mapping population was created to study the inheritance of the gene(s) underlying the putative Pg-NHR of B. thunbergii. To support this work, necessary genetic and genomic resources were developed, including genetic linkage maps for the two parental species (B. thunbergii and B. vulgaris) and a chromosome-scale reference genome for $B$. thunbergii. This study not only establishes foundational resources for the Berberis- $P g$ pathosystem but also demonstrates their use in an initial dissection of $P g$-NHR, with the long-term hope of contributing insight into possible novel mechanisms of durable resistance to the stem rust pathogen.

\section{Results}

Variant detection and linkage map construction

Genotyping-by-sequencing (GBS) libraries were constructed for the two parental lines (B. vulgaris accession 


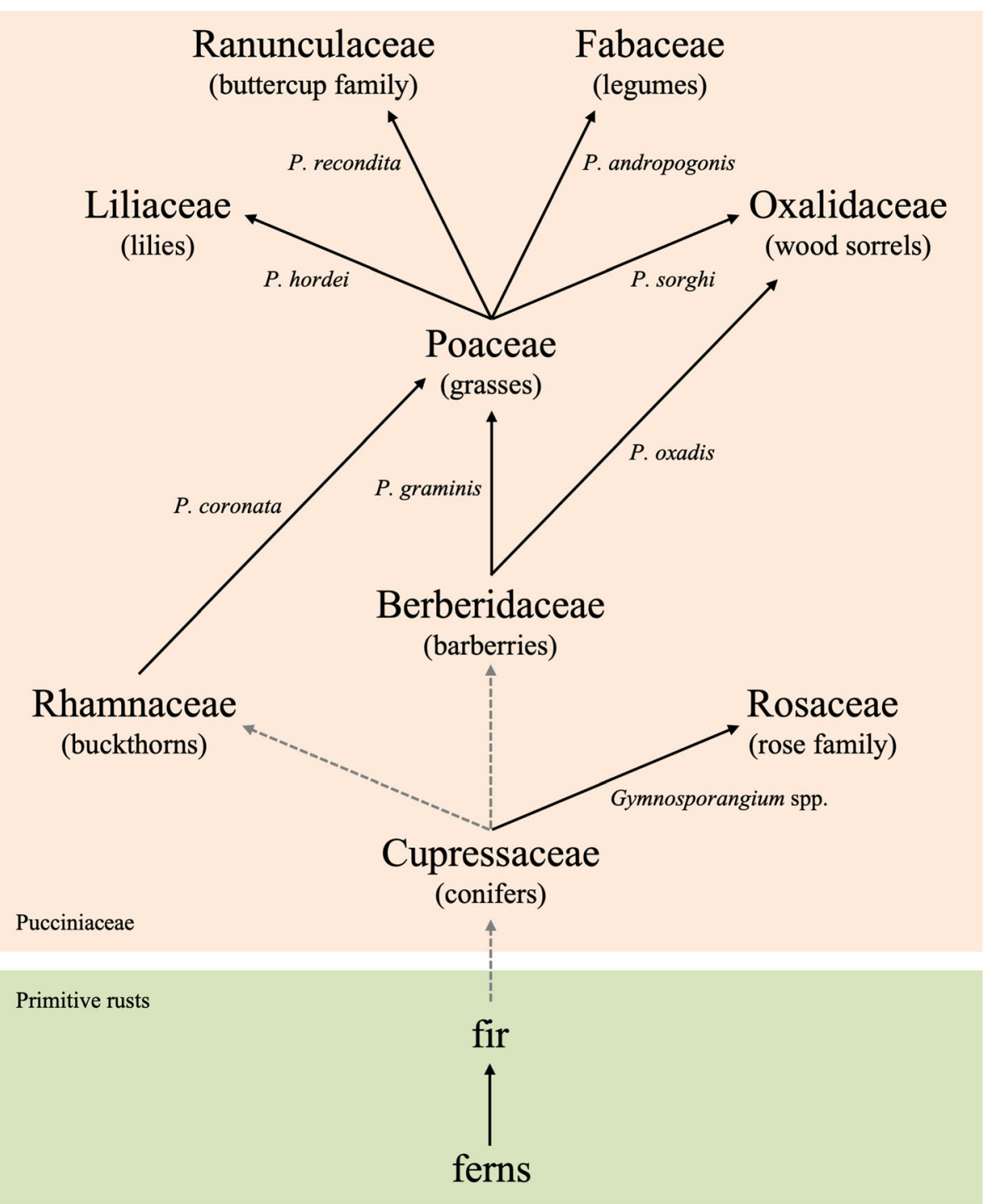

Fig. 1 Schematic of the hypothesized evolution of modern day macrocyclic, heteroecious Puccinia species. Host jump is believed to be a frequent event in the evolution of rusts, and barberries are thought to be one of the first eudicots parasitized by the rusts. Dotted arrows indicate a lack of existing rust species connecting the respective host groups. In such cases, connection is indirectly supported by relative morphological similarity among teliospores. Solid arrows connect two host species between which the indicated rust species alternate

'Wagon Hill' and B. thunbergii accession 'BtUCONN1') and their 182 interspecific $B$. xottawensis $F_{1}$ progeny, generating a total of $60 \mathrm{~Gb}$ of data [ 401 million 150bp paired end (PE) reads]. After quality parsing and demultiplexing, an average of 3 million high quality reads per genotype were retained by the GBS-SNPCROP pipeline [38] (Additional file 1). Using the high quality reads from the two parents, a mock reference (MR) comprised of 87,089 centroids (i.e. consensus GBS fragments) was generated, comprising a total length of approximately $15.4 \mathrm{Mbp}$.

A total of 15,411 polymorphic markers, including 14,043 SNPs (average depth $\mathrm{D}_{\mathrm{SNPs}}=41.5$ ) and 1368 indels $\left(D_{\text {indels }}=36.4\right)$, were identified by mapping all high-quality reads from the population to the MR. A detailed account of the winnowing of these markers via a progression of filters to obtain the final sets of markers for linkage map construction is provided in Table 1. Separate genetic linkage maps were constructed for each parental species, using a two-way pseudo-testcross mapping strategy [39]. After culling individual $F_{1}$ progeny with $>30 \%$ missing data, 161 and 162 individuals were retained for $B$. thunbergii and $B$. vulgaris linkage map construction, respectively. The $B$. thunbergii map was constructed using a total of 1757 markers (1497 and 260 from Marker Sets 1 and 2, respectively; see Table 1 ), and the $B$. vulgaris map was constructed using a total of 706 markers 
Table 1 Description of the sequence of filters applied to obtain the final marker sets for linkage map construction

\begin{tabular}{|c|c|c|}
\hline Filter descriptions, in order of application & Markers removed at each step & Markers retained \\
\hline 1. More than $30 \%$ missing genotype calls across the population ${ }^{a}$ & 6106 & 9305 \\
\hline 2. Heterozygous in both parents & 272 & 9033 \\
\hline 3. Homozygous for alternate alleles in the two parents ${ }^{b}$ & 3982 & 5051 \\
\hline 4. Deviates significantly from expected allele depth ratio in heterozygotes ${ }^{c}$ & 1801 & 3250 \\
\hline 5. Segregating genotypes unsupported by parental genotypes ${ }^{d}$ & 697 & 2553 \\
\hline 6. Deviates significantly from expected Mendelian segregation ${ }^{e}$ & 90 & 2463 \\
\hline Final markers for the $B$. thunbergii linkage map $(B t \times B v)$ & & 1757 \\
\hline Marker Set 1: ab × aa & & 1497 \\
\hline Marker Set 2: cd X-- & & 260 \\
\hline Final markers for the $B$. vulgaris linkage map $(B t \times B v)$ & & 706 \\
\hline Marker Set 3: ee $\times$ ef & & 600 \\
\hline Marker Set 4: -- × gh & & 106 \\
\hline
\end{tabular}

a This first filter was applied to the initial set of 15,411 markers (SNPs and indels) identified by the GBS-SNP-CROP pipeline

${ }^{b}$ If both parents are homozygous for the marker, no variation will be observable among the progeny (i.e. all $F_{1}$ progeny will be heterozygous for the marker)

c Mean allele depth ratio across heterozygous $F_{1}$ progeny deviates $>25 \%$ from the expected bi-allelic depth ratio of 1:1

d Lack of parental genotypes (missing data) and/or parental genotyping errors can prevent the unique assignment of gametic origin. For example, while ab $\times$ aa is expected to segregate only as aa and ab among the progeny, the alternate homozygote (bb) may be observed due to parental genotyping error. All such markers were removed from the analysis

e Segregation ratio of genotypes deviates more than two standard deviations from the expectation for each marker set; such markers were removed due to their high segregation distortion

(600 and 106 from Marker Sets 3 and 4, respectively). For both parental species, the remaining markers coalesced into 14 distinct linkage groups, in agreement with the reported chromosomal number in these Berberis spp. (Additional file 2: Figure S1).
Summary statistics of the two genetic linkage maps are detailed in Table 2. The B. thunbergii map consists of 598 recombination bins (i.e. mapped loci) and has a total length of $1474 \mathrm{cM}$. The numbers of bins in each of the 14 linkage groups (LGs) range from 23 (LG14) to 60

Table 2 Comparative summary statistics of the genetic linkage maps for B. thunbergii accession 'BtUCONN1' (Bt) and B. vulgaris accession 'Wagon Hill' (Bv)

\begin{tabular}{|c|c|c|c|c|c|c|c|c|}
\hline \multirow{2}{*}{$\begin{array}{l}\text { Linkage } \\
\text { Groupa }\end{array}$} & \multicolumn{2}{|c|}{ Length (cM) } & \multicolumn{2}{|c|}{ Number of markers } & \multicolumn{2}{|c|}{ Number of loci } & \multicolumn{2}{|c|}{ Mean distance between loci (cM) } \\
\hline & $B t$ & $B V$ & $B t$ & $B V$ & $B t$ & $B V$ & $B t$ & $B V$ \\
\hline 1 & 122.1 & 145.5 & 178 & 74 & 60 & 37 & 2.1 & 4.0 \\
\hline 2 & 98.1 & 130.4 & 122 & 63 & 41 & 31 & 2.5 & 4.3 \\
\hline 3 & 119.7 & 134.1 & 140 & 58 & 50 & 28 & 2.4 & 5.0 \\
\hline 4 & 110.8 & 154.1 & 139 & 44 & 48 & 28 & 2.4 & 5.7 \\
\hline 5 & 101.6 & 143.0 & 109 & 54 & 47 & 27 & 2.2 & 5.5 \\
\hline 6 & 115.7 & 135.0 & 195 & 76 & 49 & 31 & 2.4 & 4.5 \\
\hline 7 & 112.0 & 121.4 & 151 & 33 & 50 & 21 & 2.3 & 6.1 \\
\hline 8 & 102.0 & 120.2 & 145 & 44 & 34 & 26 & 3.1 & 4.8 \\
\hline 9 & 96.8 & 134.6 & 148 & 63 & 46 & 32 & 2.2 & 4.3 \\
\hline 10 & 93.0 & 121.2 & 88 & 25 & 34 & 14 & 2.8 & 9.3 \\
\hline 11 & 87.4 & 64.6 & 110 & 39 & 43 & 21 & 2.1 & 3.2 \\
\hline 12 & 118.3 & 86.8 & 75 & 35 & 39 & 15 & 3.1 & 6.2 \\
\hline 13 & 101.0 & 139.7 & 100 & 52 & 34 & 23 & 3.1 & 6.3 \\
\hline 14 & 95.9 & 83.3 & 55 & 32 & 23 & 13 & 4.4 & 6.9 \\
\hline Average & 105.3 & 122.4 & 125.4 & 49.4 & 42.7 & 24.8 & 2.6 & 5.5 \\
\hline
\end{tabular}

${ }^{a}$ Linkage group designations (1-14) are based on the $B$. thunbergii cv. 'Kobold' genome assembly. Linkage Group 1 anchors to the longest pseudo-molecule in the Kobold assembly (99.76 Mbp); Linkage Group 2 to the next longest (99.56 Mbp); as so on to Linkage Group 14 (54.72 Mbp) (see Additional file 2: Table S2) 
(LG2), with an average distance between adjacent bins of $2.6 \mathrm{cM}$. In comparison, the B. vulgaris map consists of 347 bins and a total length of $1714 \mathrm{cM}$. The numbers of bins in each of these 14 LGs range from 13 (LG14) to 37 (LG2), with an average distance between adjacent bins of $5.5 \mathrm{cM}$. Marker names, alleles, and genetic positions (cM), as well as a color-coded visualization of the recombination events within all members of the mapping population are provided in Additional file 3 ( $B$. thunbergii) and Additional file 4 (B. vulgaris).

\section{Disease phenotyping}

To determine disease responses to $P g$, the parents and all $F_{1}$ progeny were inoculated with basidiospores ejected from germinated teliospores produced by overwintered telia of $P g$ found on naturally infected Elymus repens. The progeny segregated into four clear phenotypic classes, ranging from resistant to susceptible (Fig. 2, Table 3). Disease phenotypes were successfully obtained for 153 progeny used for linkage map construction. Of those, 25 exhibited a clear resistant reaction similar to that of the B. thunbergii parent (Fig. 2c) and 61 exhibited a clear susceptible reaction similar to that of the $B$. vulgaris parent (Fig. 2f). Of the remaining 67 lines, 38 exhibited moderate resistance (Fig. 2d) and 29 exhibited moderate susceptibility (Fig. 2e).

\section{QTL analysis}

To map regions associated with $P g$-NHR in B. thunbergii, composite interval mapping (CIM) analysis was conducted using the linkage maps of both parents and the 4-point stem rust reaction type described above. Based on the LOD threshold score of 3.9 declared via permutation analysis, CIM analysis resulted in the identification of a single significant QTL (peak LOD value $=28.2$ ) located $25 \mathrm{cM}$ from the telomere of the short arm of $B$. thunbergii chromosome 3 (Fig. 3). The flanking markers for this $13 \mathrm{cM}$ QTL region, hereafter referred to as QPgr-3S, were determined via a detailed characterizations of the $F_{1}$ individuals with recombination events on either side of peak QTL marker M1128. The distal flanking marker $M 441$ is set by $P g$-resistant individual WH15-192, and the proximal flanking marker M969 is set by $P g$-resistant individual WH15-101 (Additional file 3). No significant QTL was detected in the B. vulgaris map.

Building a reference genome for B. thunbergii cv. 'Kobold' Approximately $129 \mathrm{~Gb}$ of sequence data was generated from 115 PacBio Single Molecule Real Time (SMRT) cells (P6-C4 chemistry on RS II), with an average read length of $10,409 \mathrm{bp}$ and a read length N50 of 15,021 bp (Additional file 2: Table S1). The haploid genome size of Kobold, a widespread green-leafed $B$. thunbergii ornamental cultivar, was estimated to be 1.37 Gbp based on k-mer analysis and $1.72 \mathrm{~Gb}$ based on flow cytometry (data not shown), two values which bound the previously published $B$. thunbergii haploid genome size (1C) of $1.51 \mathrm{~Gb}$ [40]. The FALCON-Unzip pipeline [41] resulted in a $1.36 \mathrm{~Gb}$ assembly consisting of 4671 primary contigs with contig length N50 of $0.67 \mathrm{Mbp}$ (Table 4). Their corresponding 7144 phased haplotigs had a total length of $0.88 \mathrm{~Gb}$, approximately $64 \%$ of the primary contig space. Further curation, in the form of chimera breaking and cryptic haplotig identification (see Materials and Methods), resulted in a final $1.23 \mathrm{Gbp}$ assembly consisting of 2698 primary contigs with contig length N50 of $0.76 \mathrm{Mbp}$ (Table 4). The number of haplotigs in the final assembly increased to 8790 , with a combined length of $0.99 \mathrm{~Gb}$ (> 80\% of the primary contig space).

Genome completeness and contamination analyses revealed a final genome assembly of acceptable quality, featuring complete representation of $80.9 \%$ of the BUSCO core plant gene set and only $15.1 \%$ missing BUSCO genes. $83.0 \%$ of the BtUCONN1 GBS fragments, $80.71 \%$ of the PacBio preads, and $92.2 \%$ of the RNA-seq data (in proper pair) aligned to the final assembly. After the initial FALCON-Unzip assembly, 119 primary contigs showed significant sequence similarity to plant cpDNA and mtDNA sequence; but this number dropped to only one primary contig in the final assembly as a result of intensive haplotig purging and curation.

The primary contigs from the final assembly were guided into chromosome-level scaffolds (pseudo-molecules) on the basis of three-dimensional proximity information obtained via chromosome conformation capture analysis (Hi-C) [42]. Of the 2698 primary contigs, 97\% (2611 contigs, $1.20 \mathrm{Gbp}$ ) successfully assembled into 14 pseudo-molecules representing the 14 chromosomes of B. thunbergii, as shown in the $\mathrm{Hi}-\mathrm{C}$ heatmap (Additional file 2: Figure S2). The remaining 3\% (156 contigs, $33.5 \mathrm{Mbp}$ ) were designated as unscaffolded contigs. Detailed summary statistics of the 14 pseudo-molecules comprising the B. thunbergii cv. 'Kobold' reference assembly can be found in Additional file 2: Table S2.

\section{Anchoring the genetic linkage maps to the physical assembly and assigning chromosome numbers}

Using BLASTn with MR centroids as queries, the positions of the mapped GBS markers within the final Hi-C assembly were used to anchor the genetic linkage maps of both parental species to the Kobold physical map. As illustrated in Fig. 4, a very high degree of synteny is observed between the two species, with co-linearity to the Kobold physical map being 95.1 and $92.9 \%$ for the B. thunbergii and B. vulgaris linkage maps, respectively. The physical positions of a small percentage of loci in both linkage maps (3.9\% in B. thunbergii and $5.1 \%$ in B. vulgaris) were ambiguous, in 



Fig. 2 Representative disease responses of the two mapping population parents and their $F_{1}$ progeny. a Resistant reaction of $B$. thunbergii accession 'BtUCONN1', showing no visual symptoms; $\mathbf{b}$ Susceptible reaction of $B$. vulgaris accession 'Wagon Hill', showing dense pycnia on the upper leaf surface and prolific, well-developed aecia on the lower surface; c Resistant reaction (score of 1 on the four-point scale) of $B$. xottawensis progeny 'WH15-039', showing sparse flecking; d Moderate resistant reaction (score 2) of B. xottawensis progeny 'WH15-063', showing evident necrotic lesions and some pycnia formation; e Moderate susceptible reaction (score 3) of B. Xottawensis progeny 'WH15-128', showing well-developed pycnia and aecia, alongside sparse necrotic lesions; and $\mathbf{f}$ Susceptible reaction (score 4) of B. xottawensis progeny 'WH15-149', showing well-developed pycnia and aecia and no evident necrosis. All photos were taken 14 days post-inoculation

Table 3 Descriptions of the disease reactions of the $B$ xottawensis progeny comprising the $F_{1}$ mapping population

\begin{tabular}{ll}
\hline Scale $^{a}$ & Description \\
\hline 1 & Sparse flecking and necrotic lesions, \\
& up to 3 tiny pycnia without aecia on a leaf \\
2 & Evident necrotic lesions; 5 to 15 obvious pycina \\
& with or without aecia on a leaf \\
3 & 5 to 15 well developed pycnia with aecia on a leaf; \\
& sparse necrotic lesions \\
4 & $>15$ well-developed pycnia and aecia; not \\
& preceded by necrosis
\end{tabular}

${ }^{a}$ A general 4-point scale was developed for phenotyping the disease reactions of the $F_{1}$ mapping population. $A$ resistant $(R)$ reaction is indicated by a score of 1 , a moderate resistant (MR) reaction by 2 , a moderate susceptible (MS) reaction by 3 , and a susceptible (S) reaction by 4 . Representative images for the four disease classes are shown in Fig. 2 that they could not be assigned to unique positions in the physical assembly. Another small percentage of loci $(0.93 \%$ in B. thunbergii and $1.12 \%$ in B. vulgaris) exhibited unambiguous BLAST hits to different chromosomes than in the linkage map, as indicated by dots in Fig. 4. The approximate centromere positions were visually inferred from the $\mathrm{Hi}-\mathrm{C}$ heatmap (Additional file 2: Figure S2).

To assign chromosome numbers to linkage groups, the pseudo-molecules from the Kobold physical assembly were sorted, longest to shortest. The linkage group (LG) that anchored to the longest pseudo-molecule in the Kobold assembly (99.76 Mbp) was designated LG1; the next longest pseudo-molecule was designated LG2 (99.56 Mbp); and so on to LG14 (54.72 Mbp) (see 




Table 4 Summary statistics of the B. thunbergii cv. 'Kobold' genome assembly, by stage

\begin{tabular}{|c|c|c|c|c|c|}
\hline \multirow[t]{2}{*}{ Variables } & \multicolumn{2}{|c|}{ FALCON-Unzip } & \multicolumn{2}{|c|}{ Final assembly ${ }^{a}$} & \multirow{2}{*}{$\begin{array}{l}\mathrm{Hi}-\mathrm{C} \\
\text { scaffolding }^{\mathrm{b}}\end{array}$} \\
\hline & $\begin{array}{l}\text { Primary } \\
\text { contigs }\end{array}$ & Haplotigs & $\begin{array}{l}\text { Primary } \\
\text { contigs }\end{array}$ & Haplotigs & \\
\hline Number of contigs & 4671 & 7144 & 2698 & 8790 & 14 \\
\hline Total length (Gbp) & 1.36 & 0.88 & 1.23 & 0.99 & 1.20 \\
\hline Longest (Mbp) & 8.60 & 1.49 & 8.60 & 1.49 & 99.76 \\
\hline Shortest (bp) & 8581 & 561 & 20,469 & 561 & 54.72 \\
\hline$>100$ kbp (\%) & $2551(54.6)$ & 2836 (39.7) & 2229 (82.6) & 3126 (35.6) & $14(100)$ \\
\hline$>1 \mathrm{Mbp}(\%)$ & $289(6.2)$ & $9(0.1)$ & $289(10.7)$ & $9(0.1)$ & $14(100)$ \\
\hline Mean length (Mbp) & 0.29 & 0.12 & 0.46 & 0.11 & 85.40 \\
\hline N50 length (Mbp) & 0.67 & 0.21 & 0.76 & 0.19 & 88.62 \\
\hline GC content (\%) & 37.6 & 37.7 & 37.7 & 37.7 & 37.66 \\
\hline
\end{tabular}

a After application of the Purge Haplotigs pipeline [61] and manual contig curation (i.e. chimera breaking and haplotig re-assignment)

${ }^{b}$ All statistics for the $\mathrm{Hi}-\mathrm{C}$ assembly refer to scaffolds rather than contigs. For details of individual pseudo-molecules, see Additional file 2: Table S2 


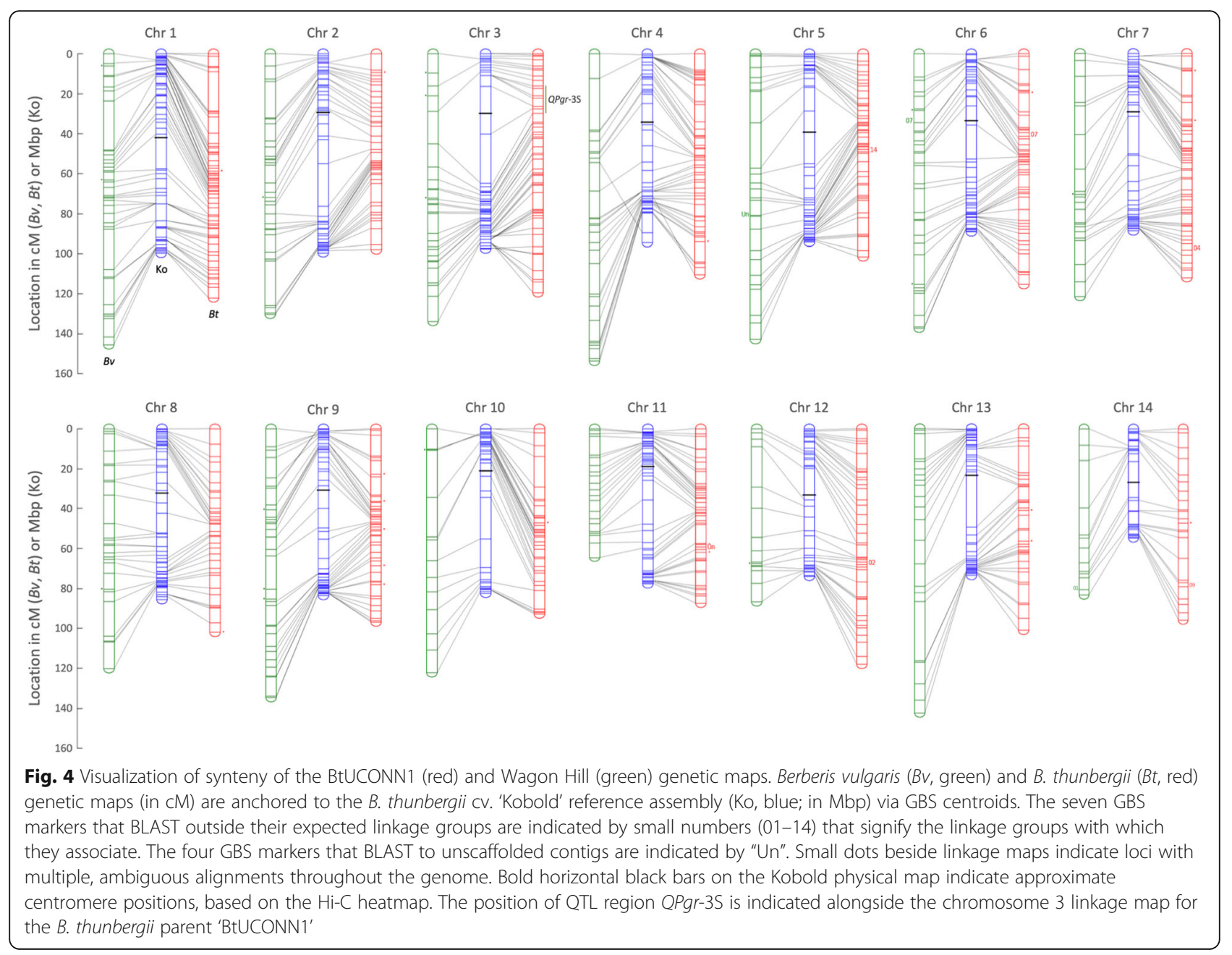

Additional file 2: Table S2). Because there was perfect agreement between the number of observed linkage groups and the expected chromosome number for the species [40], LG1 was simply re-assigned as Chromosome 1 and so on.

\section{Transcriptome assembly}

A total of $59.6 \mathrm{~Gb}$ of data, comprised of $\sim 198$ million 150 bp PE reads, was obtained by sequencing a library of 10 different tissues from the $B$. thunbergii reference accession 'Kobold', including immature leaf tissues sampled at various time points following inoculation with $P g$ (Additional file 2: Table S3). Using the Trinity pipeline [43] and the final Kobold assembly as a guide, a $189.3 \mathrm{Mbp}$ transcriptome was assembled, containing 122,872 putative transcripts and 55 , 186 cDNA sequences (complete ORFs) (see Table 5 for summary statistics). Quality and completeness of the transcriptome assembly were assessed via TransRate [44] and BUSCO analysis [45]. To date, a TransRate score of 0.22 exceeds $50 \%$ of the published de novo assembled transcriptomes deposited in the NCBI TSA [44]. In comparison, the
TransRate score of the Kobold transcriptome is 0.40 , indicating its relative quality. Completeness statistics are also acceptable, as indicated by the fact that, of the BUSCO set of 1440 core plant genes, 1286 (89.3\%) were represented in the transcriptome, of which 651 (45.2\%) were single copy and 635 (44.1\%) were duplicated.

\section{Identification of candidate genes}

The $13 \mathrm{cM}$ QPgr-3S region was found to correspond to a $5.35 \mathrm{Mbp}$ region in the physical assembly, implicating 20 contigs (length $\mathrm{N} 50=389.7 \mathrm{kbp}$ ). In an effort to refine the assembly within the QTL region, these 20 contigs were locally re-assembled using canu [46], resulting in a final set of 13 contigs with a reduced total length of 5.10 Mbp and an increased contig length N50 of $508.5 \mathrm{kbp}$. Using RepeatMasker [47], 5.6\% ( $373 \mathrm{kbp})$ of the Qpgr$3 S$ region was masked as repetitive elements using $A$. thaliana as the model. A total of 219 retroelements were found, of which 178 are LTRs (79 Ty1/Copia and 99 Gypsy/DIRS1) and 41 are LINEs (L1/CIN4). Another approximately $9 \mathrm{kbp}$ of sequence were found to 
Table 5 Descriptive statistics of the B. thunbergii cv. 'Kobold' reference-guided transcriptome assembly

\begin{tabular}{ll}
\hline Trinity reference-based assembly results & \\
\hline Number of transcripts & 122,872 \\
Total length (bp) & $189,291,041$ \\
Mean length (bp) & 1541 \\
Number of ORFs (\%) & $55,186(44.28 \%)$ \\
Transcript length N50 (bp) & 1991 \\
GC Content & $40.0 \%$ \\
& \\
TransRate results & \\
TransRate score & 0.403 \\
TransRate optimal score & 0.427 \\
TransRate cutoff & 0.037 \\
Number of good contigs (\%) & $120,972(98.5 \%)$ \\
BUSCO results & \\
Complete (\%) & 1286 (89.3\%) \\
Complete and single-copy (\%) & $651(45.2 \%)$ \\
Complete and duplicated (\%) & $635(44.1 \%)$ \\
Fragmented (\%) & $47(3.3 \%)$ \\
Missing (\%) & $107(7.4 \%)$ \\
\hline
\end{tabular}

correspond to DNA transposons. Regions of simple sequence repeats occupy a total length of $130 \mathrm{kbp}$, and 32 small RNAs were found.

Functional annotation of the QPgr-3S region resulted in the identification of 576 high confidence $(\mathrm{HC})$ genes. Of these, 450 were annotated based on the reference transcriptome (evidence-based) and 126 were annotated based on gene prediction models (ab initio). To help identify a short list of candidate genes potentially associated with $P g$-NHR and prioritized for ongoing investigation, the list of $\mathrm{HC}$ genes was cross-referenced to the results of two other analyses: Differential gene expression (DGE) and presence/absence analysis (see Materials and Methods). Time course DGE analysis led to the identification of five genes (TR27614, TR9306, TR20791, TR5393, and TR12856) that express differentially under $\mathrm{Pg}$ inoculation (Additional file 2: Figures S3 and S4). Genes TR27614 and TR9306 exhibit a similar pattern of gradual down-regulation starting around $48 \mathrm{~h}$ postinoculation (hpi). Gene TR20791 exhibits up-regulation during the first $48 \mathrm{hpi}$, followed by down regulation after 72 hpi. In contrast, genes TR5339 and TR12856 appear initially down-regulated before gradually climbing back to their original levels after 72 hpi. Presence/absence analysis identified two genes that are present in the $B$. thunbergii reference but appear to be either completely absent (MA26) or are missing whole exons (MA262) in B. vulgaris (Additional file 2: Figure S5). The evidence for possible absence in B. vulgaris is particularly strong with MA026 due to the high coverage of $B$. vulgaris reads in the immediate vicinity of the gene (Additional file 2: Figure S5).

Combined with the linkage evidence from the QTL analysis, the results of the time course DGE and presence/absence analyses elevate the seven genes identified above to a status of candidate genes associated with $P g$-NHR. As such, these candidates were selected for detailed functional annotation; and orthologous sequences were found for three of them (TR20791, TR27614, and TR12856) in the UNIPROT and Phytozome databases. Specifically, gene TR20791 is associated with a dormancy-related auxin repressor protein family; TR27614 exhibits high sequence similarity with zinc finger DNA-binding proteins; and TR12856 belongs to the glutamine synthetase (glutamateammonia ligase activity) protein family (Additional file 5). The other four candidate genes had no hits in any public database used for functional annotation and thus are potentially Berberis-specific genes, or at the very least are novel genes previously uncharacterized in other species. As the application of next-generation sequencing has become routine in genomic studies, identification of high numbers of completely novel transcripts has been found to be common in both model and non-model species (e.g. see $[48-51])$.

\section{Discussion}

\section{Genetic and genomic resource development}

Familiar, commonly used mapping populations for genetic linkage map construction in plants include segregating $F_{2}$ lines, backcross populations, doubled haploids, and recombinant inbred lines. In self-incompatible perennial plant species, however, particularly those with long generation times like barberries, such typical mapping populations are difficult, if not impossible, to produce. To overcome such challenges, the so-called "pseudo-testcross" strategy was first proposed by Grattapaglia and Sederoff (1994) and successfully applied to construct a genetic linkage map in forest trees [39]. According to this strategy, a mapping population of full-sib $F_{1}$ progeny is developed by crossing two unrelated and highly heterozygous (i.e. not inbred) individuals. Gametic recombinations can be tracked in such a population because strategicallychosen sets of markers obey the segregation patterns found in typical testcrosses. The strategy has been widely used in plant species for which other approaches are unsuitable [52-54].

In this study, using a pseudo-testcross strategy, genetic linkage maps were developed for both $B$. thunbergii and $B$. vulgaris from a single interspecific $F_{1}$ mapping population. As a result of the stringent quality filters applied to the set of de novo GBS markers used, nearly $100 \%$ of the markers were placed successfully in the linkage maps 
of the two species. Although flow cytometry analysis indicates comparable genome sizes between the two parents (B. thunbergii: $1.72 \mathrm{Gbp}$; B. vulgaris: $1.69 \mathrm{Gbp}$ ), the total length of the BtUCONN1 (B. thunbergii) linkage map obtained in this study is roughly $15 \%$ smaller than that of the Wagon Hill (B. vulgaris) map (1474 cM vs. $1714 \mathrm{cM})$. This incongruity with the expected differences in physical genome sizes is likely due to the significantly fewer markers available for the $B$. vulgaris map as compared to those available for $B$. thunbergii (706 vs. 1757). Low marker density often results in inflated genetic distances [55], so it is expected that additional markers would reduce the overall length of $B$. vulgaris linkage map. The significantly lower number of markers available for $B$. vulgaris is likely a result of the relatively lower level of diversity observed in this species as a result of the severe genetic bottleneck presumed during its colonial introduction from Europe into North America [21].

The two linkage maps developed in this study are the first for any species within the plant order Ranunculales. The relatively even distribution of markers across the 14 chromosomes of both species permits initial QTL analysis of acceptable resolution, with approximately 87 and 65\% of the inter-marker distances being less than $5 \mathrm{cM}$ for $B$. thunbergii and $B$. vulgaris, respectively. In addition, the strong synteny observed between the two independent maps is strong evidence of their reliability (Fig. 4).

As a complement to genetic resources like mapping populations and linkage maps, a high-quality reference genome can serve as an invaluable resource in dissecting QTLs, identifying underlying candidate genes, and facilitating their detailed characterization. In this study, contemporary sequencing and scaffolding technologies were used to develop a highly contiguous de novo reference genome for B. thunbergii. Using PacBio SMRT sequencing and chromosome conformation capture data, a $1.2 \mathrm{~Gb}$ haploid assembly of $B$. thunbergii cv. 'Kobold' was successfully assembled into 14 chromosome-scale pseudomolecules. As with the linkage maps, this reference is the first of its kind for a member of both the Berberidaceae family as well as the order Ranunculales, more broadly. Given the previous lack of molecular resources for barberries, the reference genome assembled in this study exemplifies the power of recent technologies to make rapid progress even in non-model systems and establishes a benchmark for the de novo assembly of a highly heterozygous plant species with a moderately sized genome.

In conclusion, the development of foundational genetic and genomic resources, including a genotyped interspecific mapping population, linkage maps for its two parental species, a chromosome-scale reference genome, and a multiple-tissue transcriptome establishes Berberis spp. as a viable research model for studying $P g$-NHR. Furthermore, such resources promise to facilitate related endeavors, including global rust surveillance work and ornamental horticulture breeding.

\section{QPgr-3S and the identification of candidate genes for Pg- NHR}

The long-term goal of this research is to identify candidate gene(s) governing $P g-\mathrm{NHR}$ in B. thunbergii. As an initial step in that direction, the genetic and genomic resources developed here enabled the identification of a single QTL of large effect (LOD > 28) on the short arm of B. thunbergii chromosome 3 (Fig. 3). This $13 \mathrm{cM}$ QTL region, dubbed Qpgr-3S, was found to span 13 physical contigs and contain a total of 576 high-confidence genes. Of these, seven were short-listed as relatively high priority candidate genes for follow up studies, including three exhibiting homology to genes in public databases, including dormancy-associated auxin repressor proteins (TR20791), zinc ion binding proteins (TR27614), and glutamine synthetase proteins (TR12856).

The current model of disease resistance suggests that plant immune responses can be grouped broadly into two major classes, namely pre-invasion defense triggered by pathogen-associated molecular patterns (PAMP-triggered immunity) and post-invasion defense triggered by pathogen effectors (effector-triggered immunity) [56, 57], both of which have been shown to implicate a wide range of defense-related proteins. Three of the seven candidate genes identified here in this study exhibit homology to gene families implicated in disease resistance in the literature. For example, auxin is known to function as a modulator of salicylic acid, a phyto-hormone essential to the induction of systemic acquired resistance in plants [58]; zinc finger transcription factors have been implicated in the regulation of a gene affecting rust germ tube differentiation [59]; and glutamine synthetase proteins are known to play key roles in plant defense against pathogens via amino acid metabolism [60].

The identification of both the QPgr-3S region and a set of high-priority candidate genes demonstrates the utility of the genetic and genomic resources developed in the study to probe the genes underlying $P g$-NHR exhibited by B. thunbergii. Such results, however, are but the first step toward identifying the genes governing Pg-NHR; and further work is required to validate and dissect the QTL region, in addition to testing candidate gene hypotheses.

\section{Possible modes of inheritance of $\mathrm{Pg}$-NHR}

From the practical standpoint of breeding for improved resistance to wheat stem rust, the central questions regarding $P g$-NHR concern the nature and modes of inheritance of the underlying genes. As previously observed in a natural interspecific barberry hybrid population [21], $\mathrm{F}_{1}$ interspecific hybrids exhibit a range of reactions to $P g$, from fully resistant to fully susceptible, with various 
intermediate forms. This range of reactions was similarly observed in the $F_{1}$ mapping population developed for this study (Fig. 2c-f and Table 3). If one assumes that the $P g$-resistance in $B$. thunbergii is governed by a single gene, independent assortment during meioses would invariably result in homozygous $P g$-susceptible $B$. thunbergii progeny. To date, however, no accession of B. thunbergii has exhibited such susceptibility, despite extensive investigation (see Background); thus a single gene governing the $P g$-resistance in $B$. thunbergii is unlikely. Polygenic NHR has been suggested in other studies as well, including rice NHR to wheat stem rust and barley NHR to powdery mildews, oat stem rust, and other non-adapted rust species [19, 61, 62].

If indeed the QPgr-3S region plays a role in Pg-NHR, the data suggest that its underlying gene(s) are necessary but not sufficient for resistance. In other words, this study at most provides a first insight into a larger gene network regulating $P g$-NHR in $B$. thunbergii. Indeed, in light of the lack of segregation in the non-host parental species B. thunbergii, the segregation of resistance among $F_{1}$ hybrids suggests the possible existence of some critical gene(s), by definition fixed within the $B$. thunbergii genepool, upstream of QPgr-3S. Because of their fixed state within $B$. thunbergii, such gene(s) cannot be mapped in an $F_{1}$ population; but if recessive, their single dosage in an $\mathrm{F}_{1}$ would permit susceptibility to $P g$, thus allowing the detection of background resistance genes (e.g. QPgr-3S). In all likelihood, $Q P g r-3 S$ is not a critical region conferring $P g$-NHR but is rather a region contributing to $P g$ resistance. Strategic crosses among the $\mathrm{F}_{1}$ progeny and/or backcrosses to $B$. thunbergii will be necessary to test this hypothesis and identify those critical gene(s) regulating Pg-NHR in B. thunbergii, work shown to be feasible by the current study.

\section{Conclusions}

In this paper, we report the development of publiclyavailable foundational genetic and genomic resources for the novel Berberis-Pg pathosystem, including the first genetic maps for two Berberis species (B. thunbergii and $B$. vulgaris), a chromosome-scale reference genome for $B$. thunbergii, and a related transcriptome to facilitate the characterization of genetic mechanism(s) of $P g-\mathrm{NHR}$. Future work should focus on the validation, further characterization, and dissection of the identified QTL, including testing of candidate gene hypotheses. Beyond this, now that the Berberis-Pg pathosystem has been shown to be a viable means of probing the mechanism of $P g$-NHR in B. thunbergii, future work must also wrestle with the significant question of potential translatability of such resistance to wheat. Such translatability is certainly not a given, particularly in light of the fact that the infecting spores are different for Berberis (basidiospores) and grass (urediniospores) hosts. However, because the two life stages in question belong to the same pathogenic organism and because Berberis is the likely ancestral host of that organism prior to its host expansion to the grasses (see Background), the possibility exists that the mechanism of $P g-\mathrm{NHR}$ in $B$. thunbergii may provide relevant insight into breeding durable resistance in wheat. With this study, the foundation is laid to eventually answer this question.

\section{Methods \\ Mapping population development}

A B. xottawensis mapping population consisting of 182 $F_{1}$ individuals was derived from an interspecific cross between $B$. thunbergii accession 'BtUCONN1' (pollen parent) and B. vulgaris accession 'Wagon Hill' (female parent). True to its species, BtUCONN1 is a non-host to the stem rust pathogen and is a small shrub $(0.5-2.5 \mathrm{~m}$ tall) that displays $1.3-3.8 \mathrm{~cm}$ long entire leaves and $1-2$ $\mathrm{cm}$ long inflorescences with few umbellate but mostly solitary flowers. In contrast, Wagon Hill is susceptible to stem rust and is a relatively taller shrub $(\sim 3 \mathrm{~m}$ tall $)$ that displays $2-5 \mathrm{~cm}$ long obovate to obovate-oblong leaves with highly serrated margins ( $>50$ serrations) and has 5-8 cm long pendant racemes of bright yellow flowers. The pollen parent BtUCONN1 was a feral plant maintained in the barberry collection at the research farm of the University of Connecticut (N41 ${ }^{\circ} 47^{\prime} 40.63^{\prime \prime}$, W072 $\left.13^{\prime} 39.61^{\prime \prime}\right)$, and the female parent Wagon Hill is a feral plant growing along the shoreline of the Great Bay Estuary in Durham, New Hampshire (N43.07' 30.64", W70 $\left.52^{\prime} 17.95^{\prime \prime}\right)$.

To make the interspecific cross, pollen was harvested from mature flowers of BtUCONN1 using the previously described N-pentane method [63] and stored at $4{ }^{\circ} \mathrm{C}$ until flowers of Wagon Hill reached reproductive maturity. Emasculation and hand pollination of female flowers were performed at the so-called balloon stage, when the petals begin to part slightly at the top, giving the appearance of an inflated balloon prior to opening. To break dormancy before sowing, seeds from successful crosses were stratified in wet sand in a petri dish at $4{ }^{\circ} \mathrm{C}$ for three months. Propagated cuttings of the two parents were maintained along with the $F_{1}$ mapping population in plastic pots $(11.5 \mathrm{~cm}$ diameter; $6.5 \mathrm{~cm}$ tall) filled with PRO-MIX HP growth media in the Macfarlane Greenhouse facility at the University of New Hampshire.

To verify the putative $F_{1}$ status of the individuals in the mapping population, a PCR-based species-specific marker was designed based on available GBS data [21]. A universal primer pair was designed to amplify a short genomic sequence exhibiting a length polymorphism between the two parents. Specifically, the primers (F: $5^{\prime}$ CCTGATTGGGGCTCATTATC-3'; R: 5'-AGTGAGGA 
ATTCCGAGCTGA-3') amplified a 208 bp fragment in Wagon Hill but only a 195 bp fragment in BtUCONN1, due to the presence of a $13 \mathrm{bp}$ indel (see Additional file 6: Text S1). PCR was conducted with a total reaction volume of $20 \mu \mathrm{l}(0.25 \mathrm{mM}$ of each primer, $100 \mu \mathrm{M}$ of each dNTP, $0.75 \mathrm{U}$ Taq DNA Polymerase, 10x standard Taq buffer, and $100 \mathrm{ng}$ of template DNA) subjected to the following cycling conditions: 5 mins at $94{ }^{\circ} \mathrm{C}$; $32 \mathrm{cy}-$ cles of $30 \mathrm{~s}$ at $94{ }^{\circ} \mathrm{C}, 30 \mathrm{~s}$ at $52^{\circ} \mathrm{C}$, and $15 \mathrm{~s}$ at $68^{\circ} \mathrm{C}$; and 5 mins at $68^{\circ} \mathrm{C}$. Amplified products were separated on a $3 \% \mathrm{TBE} / \mathrm{EtBr}$ agarose gel for $60 \mathrm{~min}$ at $75 \mathrm{~V}$ and imaged with UV transillumination. The $F_{1}$ status of a putative hybrid individual was considered validated if both bands from the two parental species were detected (Additional file 2: Figure S6).

\section{Genotyping and variant detection}

Genomic DNA of the 182 verified $F_{1}$ individuals and both parents was extracted from $\sim 100 \mathrm{mg}$ of lyophilized leaf tissue using a modified CTAB method [64]. Prior to GBS library preparation, isolated DNA was purified using Zymo Research's Genomic DNA Clean \& Concentrator $^{\text {rat-10 }}$ column (Catalog \# D4011), following manufacturer's protocol. Reduced representation libraries were constructed using the two-enzyme (PstI-MspI) GBS protocol described by Poland et al. [65] and sequenced via $150 \mathrm{bp}$ paired-end $(\mathrm{PE})$ reads on an Illumina HiSeq 2500 at the Hubbard Center for Genome Studies, UNH.

Raw FASTQ files were generated by CASAVA 1.8.3 and analyzed using the reference-free bioinformatics pipeline GBS-SNP-CROP [38, 66]. A Mock Reference (MR) was constructed using the high quality PE reads from the two parents; and putative variants, both SNPs and indels, were identified via alignment of high quality PE reads from the parents and all $F_{1}$ progeny to the $M R$, following the pipeline's recommended parameters for diploid species. Complete details of the GBS-SNP-CROP command lines used in this analysis, including all specified pipeline parameters, are provided in Additional file 6: Text S2.

\section{Genetic linkage map construction}

The sequence of filters applied to obtain the final sets of markers for linkage map construction is summarized in Table 1. In short, a marker was culled if it met any of the following criteria: 1) It was unscored for more than $30 \%$ of the individuals in the population; 2) It was heterozygous for both parents; 3) It failed to segregate in the population (i.e. all progeny were heterozygous for the marker); 4) Its mean ratio of primary to alternate allele depth deviated significantly from the expected ratio of 1:1; and/or 5) Its segregation ratio deviated significantly from the expected ratio of 1:1, according to its marker class. As a final filter, genotypes with $>30 \%$ missing data were removed.
Linkage analysis was performed using the $\mathrm{R}$ package ONEMAP v2.0-4 [67], and separate linkage maps were constructed for the two parents according to a two-way pseudo-testcross mapping strategy [30]. The BtUCONN1 linkage map was constructed using Marker Sets 1 and 2, while the Wagon Hill map was constructed using Marker Sets 3 and 4 (see Table 1). For each map, a two-point test was first performed for all marker pairs, using a minimum LOD score of 4 and a maximum recombination fraction of 0.25 to group markers into linkage groups (LGs). Next, markers within each LG were ordered using the 'try' algorithm within ONEMAP.

To identify potential genotyping errors, common in GBS data [68], maps were manually inspected for the presence of singletons (apparent double crossovers) [69], which were replaced with missing values. If multiple markers were found to map to the same genetic bin, a consensus of the set of markers was chosen to represent the linkage bin for final mapping iterations, which were made until no alternative orders were generated by the 'ripple.seq' function. Final map distances were calculated with the Kosambi mapping function [70], and ideograms were generated using Mapchart 2.0 [71].

\section{Stem rust disease phenotyping}

To determine disease responses, the parents and all $F_{1}$ individuals in the mapping population were inoculated with basidiospores ejected from germinated teliospores produced by $P g$ telia found on naturally-infected Elymus repens, as previously described [21]. The pollen parent BtUCONN1 exhibits the clear non-host reaction typical of B. thunbergii. In contrast, the female parent Wagon Hill exhibits the clear susceptible reaction of $B$. vulgaris, with well-developed mature aecia visible on the abaxial surfaces of leaves. Images of typical reactions of the parents and of individuals in the $F_{1}$ mapping population are presented in Fig. 2. As detailed in Table 3, a 4-point scale was developed in response to the particular segregating characteristics observed in this population. The levels of this scale are based on the following symptoms: 1) Degree of flecking; 2) Presence and intensity of necrotic lesions; and 3) Presence and density of pycnia and aecia. All plants were scored for reaction to stem rust 14 days after inoculation.

\section{QTL analysis}

QTL analysis for $P g$ resistance was performed using both the parental and maternal genetic linkage maps using the $\mathrm{R}$ package R/qtl v1.39-5 [72]. Haley-Knott regression [73] was used, based on the composite interval mapping method (CIM); and a QTL was deemed significant if its peak LOD score exceeded the threshold determined via permutation analysis (1000 permutations, 5\% significance level). 


\section{Reference genome assembly}

Due to its relevance not only to $P g$-NHR research but also to ornamental breeding, B. thunbergii cv 'Kobold', a commercial green-leafed cultivar common in the ornamental industry, was selected for whole genome sequencing. Kobold is a heterozygous diploid $(2 n=2 x=28)$ and is a non-host to stem rust [33]. Cuttings of Kobold were obtained from the barberry collection at the University of Connecticut, rooted, and maintained in the MacFarlane Greenhouses at UNH under standard conditions for barberry [21]. For sequencing, $\sim 2 \mathrm{~g}$ of fresh young leaves were collected from 4 to 6 clonally propagated plants and flash frozen in liquid nitrogen. Genomic DNA was extracted using a modified CTAB procedure [74] and quantified via both fluorometry (Qubit, Thermo Fisher Scientific, Waltham, U.S.A.) and agarose gel electrophoresis with a lambda DNA standard. A 20-kb BluePippin kit (PacBio) was used for Single Molecule Real Time (SMRT) library preparation; and 115 SMRT cells were sequenced on the PacBio RS II system at the UC Davis Genome Center, using P6-C4 chemistry. All data were collected as $6 \mathrm{~h}$ sequencing videos.

The FALCON and FALCON-Unzip toolkits (FALCONintegrate v1.8.2) [41] were used for whole genome assembly and phasing. FALCON is described as a Hierarchical Genome Assembly Process pipeline that generates a genome assembly from long PacBio reads through the following basic steps: 1) Raw read error correction via alignment of subreads; 2) Pre-assembly of long, errorcorrected reads; 3) Overlap detection of pre-assembled reads; 4) Overlap filtering; 5) Overlap graph construction; and 6) Graph-based contig construction. After this initial assembly, FALCON-Unzip is used in highly heterozygous species to resolve the distinct haplomes (i.e. to unzip the genome) based on patterns of structural variants and associated SNPs (i.e. haplotype blocks). This unzip process gives rise to a set of so-called primary contigs (the primary assembly) and a set of associated haplotigs (phased variants of the primary contigs, in regions of high heterozygosity). Complete details of the FALCON configuration file used in this study are provided in Additional file 6: Text S3. Finally, the Arrow algorithm within the 'GenomicConsensus' PacBio package (https://github.com/PacificBiosciences/GenomicConsensus) was used to polish the phased primary contigs and their associated haplotigs. Genome size was estimated using both k-mer analysis of the error-corrected PacBio reads [75] as well as propidium iodide flow cytometric analysis using Pisum sativum L. Citrad $(2 \mathrm{C}=9.09 \mathrm{pg})$ as an internal standard (BD Accuri ${ }^{\mathrm{im}}$ C6 Cytometer) [76].

Further polishing and curation of the assembly was accomplished using the Purge Haplotigs pipeline [77]. High levels of heterozygosity in some genomic regions can lead to the incorrect assignment of haplotigs as distinct primary contigs [77]. To identify such errors and correctly assign homologous contigs to the haplotig pool, the Purge Haplotigs pipeline first performs a read-depth analysis using BEDTools [78] to flag abnormally low or high coverage contigs as potential chimeras and then performs a BLAST [79] against the entire assembly to identify putative primary contigs exhibiting high homology to one another. During this process, alignment dotplots are produced, and these are manually screened to break likely chimeras, define the final set of primary contigs as the reference sequence, and assign residual syntenic contigs as haplotigs. Complete details of the Purge Haplotigs process are provided in Additional file 6: Text S4.

\section{Assessment of genome assembly quality and hi-C scaffolding}

Quality of the final curated assembly was assessed using QUAST [80], and assembly completeness was evaluated using the set of 1440 core plant genes in BUSCO v3 [45]. To identify and purge contaminant contigs, the final assembly was BLASTed to the following databases of possible contaminants: plasmid DNA (cpDNA and mtDNA) from angiosperms, the human genome (GRCh38.p7), the Escherichia coli genome (CP017100.1), and 16S and 18S rRNAs. The rRNA database was created using the SILVA project [81], and the others were created via sampling from Genbank. To further evaluate completeness, the PacBio error-corrected reads (preads), the RNA-seq data generated for transcriptome assembly (see below), and the GBS data from the BtUCONN1 parent generated for linkage mapping were also aligned to the final assembly using BLASR [82], GMAP [83], and BWA [84], respectively.

To linearly order and orient the primary contigs into chromosome-scale pseudo-molecules, a proximity-guided assembly was performed using Phase Genomics' Proximo chromosome conformation capture (Hi-C) technology [42]. Tissue processing, chromatin isolation, library preparation, sequencing, and $\mathrm{Hi}-\mathrm{C}$ analysis were performed by Phase Genomics (Seattle, WA, USA). Finally, the BtUCONN1 genetic linkage map was used to manually curate the $\mathrm{Hi}-\mathrm{C}$ assembly using JuiceBox [85], bringing independent information to guide the ordering of a set of anchor contigs in instances of ambiguity.

\section{Anchoring of the genetic linkage maps to the physical assembly}

Orthogonal sets of markers were used to build the genetic linkage maps of the two parents; thus the two maps share no markers in common, preventing a direct assessment of synteny between the two species. The physical assembly, however, presents a potential "common language" by which the two maps can be compared, provided the markers in the linkage maps can be uniquely located in (i.e. anchored to) the physical assembly. To accomplish 
this, BLASTn [86] was performed between the MR centroids (queries) and the curated assembly (subject). Using only those centroids exhibiting unique positions within the reference genome, synteny plots were generated using the Pacth function of the Matplotlib plotting library (https://matplotlib.org/index.html). The above anchoring method was also used to project the detected $P g$-NHR QTL region onto the physical map, thus permitting insight into its underlying physical sequence.

\section{Transcriptome assembly}

For transcriptome assembly, ten different tissues, including immature leaf tissue at various time points after $P g$ inoculation, were collected from a clonally propagated plant of B. thunbergii cv. 'Kobold' (Additional file 2: Table S3). Fresh tissues were flash frozen in liquid nitrogen and ground to fine powder using mortar and pestle. Total RNA was isolated using the Zymo Research RNA Clean \& Concentrator ${ }^{\text {mis }}$ kit (Catalog \# R1015), according to manufacturers' protocol. RNAseq libraries were prepared with Illumina TruSeq ${ }^{\circ}$ RNA Library Prep Kits and sequenced via 150 bp paired-end (PE) reads on an Illumina HiSeq 2500 at the Hubbard Center for Genome Studies, UNH.

CASAVA-processed raw sequences were errorcorrected using the software BFC v1.0 [87], following the recommendations of the Oyster River Protocol For Transcriptome Assembly [88]. Error-corrected reads were processed to remove Illumina adapters and gently trimmed to remove low quality reads (Phred $\leq 5$ ) using Trimmomatic v.0.33 [89]. All post-processed reads from the ten tissues were pooled, and the transcriptome was assembled using Trinity (reference-guided de novo assembly) [34]. Assembly quality was evaluated using TransRate [44], and its completeness was assessed using the set of 1440 core plant genes in BUSCO v3 [45]. In addition to providing basic summary statistics and quality metrics, TransRate provides an overall score of transcriptome contiguity based on a suite of mapping metrics; and BUSCO evaluates assembly content based on the representation of expected single copy orthologs.

\section{Identification of candidate genes}

To facilitate the identification of candidate genes that may explain the association of the detected QTL region to $P g$ response, the physical contigs spanning the QTL region were locally re-assembled using canu [46]. The re-assembled QTL region was then structurally annotated using RepeatMasker [47] and functionally annotated with the Maker pipeline [90], using both ab-initio and transcriptome-based analyses. The set of well-supported genes within the QTL region, hereafter referred to as high-confidence $(\mathrm{HC})$ genes, were defined based on Maker's Annotation Edit Distance quality metric $(\mathrm{AED}<0.7)$ as well as the requirement that the genes be non-overlapping and between 500 and 10,000 bp in length.

Combinations of approaches were taken to pare down the full set of $\mathrm{HC}$ genes to those more likely to contribute to $P g$-NHR. A differential gene expression (DGE) analysis experiment was conducted to identify genes whose levels of expression detectably change under challenge by $P g$. Three biological replicates of immature leaves were sampled from clonally propagated $B$. thunbergii cv. 'Kobold' plants at four different time points: pre-inoculation (T0) and 48,72 , and $144 \mathrm{~h}$ postinoculation (T48, T72, and T144). Total RNA was extracted, sequenced, and processed as described above. Transcript abundance was quantified using Kallisto [91], and time course analysis was performed using Sleuth [92]. Complete details of the parameters used for transcript abundance and time course analysis are provided in Additional file 6: Text S5.

To complement the above DGE analysis, $\sim 428$ million PE $100 \mathrm{bp}$ shotgun reads from the B. vulgaris parent Wagon Hill (i.e. $>30 x$ re-sequencing depth) were aligned to the QTL region in an effort to identify $\mathrm{HC}$ genes with no apparent homolog in B. vulgaris. The final list of highpriority candidate genes is composed of those $\mathrm{HC}$ genes in the QTL region that are either differentially expressed under $P g$ inoculation or have at least one complete CDS sequence absent in B. vulgaris (Additional file 2: Figure S5). Putative protein functions and Gene Ontology (GO) terms were assigned to the candidate genes using both the Phytozome v.12.1 [93] and UniProtKB [94] databases.

\section{Additional files}

Additional file 1: Sequencing details of $B$. thunbergii accession 'BtUCONN1', B. vulgaris accession 'Wagon Hill', and the interspecific F1 (B. Xottawensis) mapping population used in this study. (XLSX $20 \mathrm{~kb}$ )

Additional file 2: Supplementary Figures and Tables. Figure S1. Genetic linkage maps of $B$. thunbergii accession 'BtUCONN1' and $B$. vulgaris accession 'Wagon Hill'. Figure S2. Hi-C heat map of the scaffolded primary contigs of B. thunbergii cv. 'Kobold'. Figure S3. Venn diagrams of high-priority candidate genes identified for further investigation. Figure S4. Time course expression plots for the five candidate genes found via DGE analysis. Figure S5. Base-by-base coverage plots in B. vulgaris accession 'Wagon Hill' for the two candidate genes identified via presence-absence analysis. Figure $\mathbf{S 6}$. Gel image of the marker used to validate the hybrid status of the individuals in the $F_{1}$ mapping population. Table S1. Summary of the raw PacBio data obtained for B. thunbergii cv. 'Kobold'. Table S2. Summary statistics of the 14 pseudo-molecules of the B. thunbergii cv. 'Kobold' reference assembly. Table S3. Details of the library of ten tissues from $B$. thunbergii cv. 'Kobold' used for transcriptome assembly. (PDF $5620 \mathrm{~kb}$ )

Additional file 3: Linkage map of B. thunbergii accession 'BtUCONN1' and associated information. (XLSX $412 \mathrm{~kb}$ )

Additional file 4: Linkage map of B. vulgaris accession 'Wagon Hill' and associated information. (XLSX $251 \mathrm{~kb}$ )

Additional file 5: MAKER features and detailed functional annotation for the seven candidate genes. (XLSX $13 \mathrm{~kb}$ ) 
Additional file 6: Supplementary Text. Text S1. Cluster sequences and primer information for the PCR-based markers used to validate the $F_{1}$ status of the individuals comprising the B. Xottawensis mapping population. Text S2. Detailed record of the GBS-SNP-CROP command lines used in this study. Text S3. Complete details of the FALCON assembly parameters used in this study. Text S4. Complete details of the script used for purging haplotigs. Text S5. Complete details of parameters used for quantifying transcripts and the sleuth $\mathrm{R}$ code for the time course analysis. (PDF $128 \mathrm{~kb}$ )

\section{Abbreviations}

CIM: Composite interval mapping; DGE: Differential gene expression; GBS: Genotyping-by-sequencing; HC genes: High confidence genes; Hpi: Hours post inoculation; LG: Linkage group; MR: Mock reference; NHR: Non-host resistance; PAMP: Pathogen-associated molecular patterns; PE: Paired end; Pg: Puccinia graminis; SMRT: Single Molecule Real Time

\section{Acknowledgements}

We thank L. Hydock for greenhouse support; M. Brand for providing pollen of BtUCONN1 and instruction in barberry pollination and propagation; T. Davis for critical feedback; M. Shields for assistance with flow cytometry; and M. Macmanes for guidance in reference and transcriptome assembly. We also wish to thank the handling editor and anonymous reviewers for their critical feedback on an earlier version of this manuscript.

\section{Authors' contributions}

$\mathrm{IH}$ conceptualized the research. $\mathrm{IH}$ and RB designed experiments and coordinated all aspects of the work. RB, AM, and IH performed the data analysis. SK contributed to the reference genome assembly and evaluation. $\mathrm{RB}, \mathrm{AM}, \mathrm{YJ}$, and IH drafted the manuscript; all authors revised the manuscript and approved its final form.

\section{Funding}

This work was supported by the Bill \& Melinda Gates Foundation [OPPGD1389, OPP1133199]; and USDA NIFA Hatch Multistate Project NE009 [NH00611-R]. Partial funding was provided by the New Hampshire Agricultural Experiment Station (Scientific Contribution Number 8203).

\section{Availability of data and materials}

All raw sequence data and final assemblies (genome and transcriptome) are available through the NCBI database. The parsed, high-quality GBS data generated for the two parental lines and the $182 \mathrm{~F}_{1}$ progeny are available through the NCBI Short Read Archive, with SRA ID's provided in Additional file 1. RNAseq data from the ten B. thunbergii cv. 'Kobold' tissues used for DGE and transcriptome assembly are linked to NCBI BioProject PRJNA478022; the assembled transcriptome itself is available under TSA ID GGRA00000000. Data related to the Kobold genome assembly, including the FALCON-Unzip primary contigs and haplotigs, the final $\mathrm{Hi}-\mathrm{C}$ guided chromosome-level assembly, and all unscaffolded contigs, are deposited in NCBI under BioProject accession number PRJNA478403. The Kobold genome is also available for visualization and annotation through the UNH WebApollo Genome Browser at http://genome.sr.unh.edu/jbrowse.

\section{Ethics approval and consent to participate}

Not applicable.

\section{Consent for publication}

Not applicable.

\section{Competing interests}

SK is a full-time employee at Pacific Biosciences, a company developing single-molecule sequencing technologies.

\section{Author details}

'Department of Molecular, Cellular, and Biomedical Sciences, University of New Hampshire, Durham, NH 03824, USA. ²Department of Agriculture, Nutrition, and Food Systems, University of New Hampshire, Durham, NH 03824, USA. ${ }^{3}$ Pacific Biosciences, Menlo Park, CA 94025, USA. ${ }^{4}$ USDA-ARS Cereal Disease Laboratory, St. Paul, MN 55108, USA.
Received: 18 February 2019 Accepted: 19 June 2019

Published online: 16 July 2019

\section{References}

1. Leonard KJ, Szabo LJ. Stem rust of small grains and grasses caused by Puccinia graminis. Mol Plant Pathol. 2005;6:99-111.

2. Singh RP, Hodson DP, Jin Y, Lagudah ES, Ayliffe MA, Bhavani S, et al. Emergence and spread of new races of wheat stem rust fungus: continued threat to food security and prospects of genetic control. Phytopathology. 2015;105:872-84.

3. Singh RP, Hodson DP, Huerta-Espino J, Jin Y, Njau P, Wanyera R, et al. Will stem rust destroy the world's wheat crop? Adv Agron. 2008;98: 271-309.

4. Peterson PD, Leonard K, Roelfs AP, Sutton TB. Effect of barberry eradication on changes in populations of Puccinia graminis in Minnesota. Plant Dis. 2005;89:935-40.

5. Jin Y, Szabo LJ, Pretorius ZA, Singh RP, Ward R, Fetch T Jr. Detection of virulence to resistance gene Sr24 within race TTKS of Puccinia graminis f. sp. tritici. Plant Dis. 2008;92:923-6.

6. Pretorius ZA, Singh RP, Wagoire WW, Payne TS. Detection of virulence to wheat stem rust resistance gene Sr37 in Puccinia graminis. f. Sp. tritici in Uganda. Plant Dis. 2000;84:203.

7. Singh RP, Hodson DP, Huerta-Espino J, Jin Y, Bhavani S, Njau P, et al. The emergence of $U g 99$ races of the stem rust fungus is a threat to world wheat production. Annu Rev Phytopathol. 2011;49:465-81.

8. Lewis CM, Persoons A, Bebber DP, Kigathi RN, Maintz J, Findlay K, et al. Potential for re-emergence of wheat stem rust in the United Kingdom. Commun Biol. 2018;1:13

9. Ayliffe $M$, Singh R, Lagudah E. Durable resistance to wheat stem rust needed. Curr Opin Plant Biol. 2008;11:187-92.

10. Lipka U, Fuchs R, Kuhns C, Petutschnig E, Lipka V. Live and let dieArabidopsis nonhost resistance to powdery mildews. Eur J Cell Biol. 2010:89:194-9.

11. Thordal-Christensen $\mathrm{H}$. Fresh insights into processes of nonhost resistance Curr Opin Plant Biol. 2003;6:351-7.

12. Mysore KS, Ryu C-M. Nonhost resistance: how much do we know? Trends Plant Sci. 2004;9:97-104.

13. Ayliffe M, Devilla R, Mago R, White R, Talbot M, Pryor A, et al. Nonhost resistance of rice to rust pathogens. Mol Plant-Microbe Interact. 2011;24:1143-55.

14. Cheng Y, Zhang H, Yao J, Wang X, Xu J, Han Q, et al. Characterization of non-host resistance in broad bean to the wheat stripe rust pathogen. BMC Plant Biol. 2012;12:96.

15. Cheng Y, Zhang H, Yao J, Han Q, Wang X, Huang L, et al. Cytological and molecular characterization of non-host resistance in Arabidopsis thaliana against wheat stripe rust. Plant Physiol Biochem. 2013;62:11-8.

16. An T, Cai Y, Zhao S, Zhou J, Song B, Bux H, et al. Brachypodium distachyon T-DNA insertion lines: a model pathosystem to study nonhost resistance to wheat stripe rust. Sci Rep. 2016;6:25510.

17. Dawson AM, Ferguson JN, Gardiner M, Green P, Hubbard A, Moscou MJ. Isolation and fine mapping of Rps6: an intermediate host resistance gene in barley to wheat stripe rust. Theor Appl Genet. 2016;129:831-43.

18. Li K, Hegarty J, Zhang C, Wan A, Wu J, Guedira GB, et al. Fine mapping of barley locus Rps6 conferring resistance to wheat stripe rust. Theor Appl Genet. 2016;129:845-59.

19. Dracatos PM, Ayliffe M, Khatkar MS, Fetch T Jr, Singh D, Park RF. Inheritance of prehaustorial resistance to Puccinia graminis f. sp. avenae in barley (Hordeum vulgare L.). Mol Plant-Microbe Interact. 2014;27:1253-62.

20. Figueroa M, Alderman S, Garvin DF, Pfender WF. Infection of Brachypodium distachyon by formae speciales of Puccinia graminis: early infection events and host-pathogen incompatibility. PLoS One. 2013. https://doi.org/10.1371/ journal.pone.0056857.

21. Bartaula R, Melo AT, Connolly BA, Jin Y, Hale I. An interspecific barberry hybrid enables genetic dissection of non-host resistance to the stem rust pathogen Puccinia graminis. J Exp Bot. 2018;69:2483-93.

22. Jin Y. Role of Berberis spp. as alternate hosts in generating new races of Puccinia graminis and P. striiformis. Euphytica. 2011;179:105-8.

23. Zhao J, Wang L, Wang Z, Chen X, Zhang H, Yao J, et al. Identification of eighteen Berberis species as alternate hosts of Puccinia striiformis f. sp. tritici and virulence variation in the pathogen isolates from natural infection of barberry plants in China. Phytopathology. 2013;103:927-34. 
24. Peterson PD. "The Barberry or Bread": The Public Campaign to Eradicate Common Barberry in the United States in the Early 20th Century. Available from: https://www.apsnet.org/edcenter/apsnetfeatures/Pages/Barberry.aspx

25. Levine MN, Cotter RU. Susceptibility and resistance of Berberis and related genera to Puccinia graminis: United States Department of Agriculture; 1932. Report No.: 300

26. Roelfs AP, Bushnell WR. Diseases, Distribution, Epidemiology, and Control. In: The Cereal Rusts. Orlando: Academic; 1985. p. 7-9.

27. Department of Agriculture. Black Stem Rust; Identification Requirements and Addition of Rust Resistant Varieties. https://www.ars.usda.gov/ARSUserFiles/ 50620500/Barberry/fedreg67-36.pdf. Accessed 28 May 2019.

28. Department of Agriculture. Black Stem Rust; Identification Requirements and Addition of Rust Resistant Varieties. https://www.ars.usda.gov/ARSUserFiles/ 50620500/Barberry/fedreg71-23.pdf. Accessed 28 May 2019.

29. Department of Agriculture. Black Stem Rust; Identification Requirements and Addition of Rust Resistant Varieties. https://www.ars.usda.gov/ARSUserFiles/ 50620500/Barberry/2007\%20fedreg72-112.pdf. Accessed 28 May 2019.

30. Department of Agriculture. Black Stem Rust; Identification Requirements and Addition of Rust Resistant Varieties. https://www.ars.usda.gov/ARSUserFiles/ 50620500/Barberry/2010\%20fedreg75-100.pdf. Accessed 28 May 2019.

31. Department of Agriculture. Black Stem Rust; Identification Requirements and Addition of Rust Resistant Varieties. https://www.ars.usda.gov/ARSUserFiles/ 50620500/Barberry/2010\%20fedreg75-173.pdf. Accessed 28 May 2019.

32. Department of Agriculture. Black Stem Rust; Identification Requirements and Addition of Rust Resistant Varieties. https://www.govinfo.gov/content/pkg/ FR-2013-05-13/pdf/2013-11318.pdf. Accessed 28 May 2019.

33. Department of Agriculture. Black Stem Rust; Identification Requirements and Addition of Rust Resistant Varieties. https://www.govinfo.gov/content/pkg/ FR-2017-09-05/pdf/2017-18712.pdf. Accessed 28 May 2019.

34. Hale IL, Connolly BA, Bartaula R. The occurrence of hybrid barberry, Berberis xottawensis (Berberidaceae), in New Hampshire and Rhode Island. Rhodora. 2015;117:384-7.

35. Arthur JC, Cummins GB. Manual of the rusts in United States and Canada. Lafayette: Purdue Research Foundation; 1934.

36. Li Y-L, Kvaček Z, Ferguson DK, Wang Y-F, Li C-S, Yang J, et al. The fossil record of Berberis (Berberidaceae) from the Palaeocene of NE China and interpretations of the evolution and phytogeography of the genus. Rev Palaeobot Palynol. 2010;160:10-31.

37. Bettgenhaeuser J, Gilbert B, Ayliffe M, Moscou MJ. Nonhost resistance to rust pathogens-a continuation of continua. Front Plant Sci. 2014;5:664.

38. Melo AT, Bartaula R, Hale I. GBS-SNP-CROP: a reference-optional pipeline for SNP discovery and plant germplasm characterization using variable length, paired-end genotyping-by-sequencing data. BMC Bioinform. 2016. https:// doi.org/10.1186/s12859-016-0879-y.

39. Grattapaglia D, Sederoff R. Genetic linkage maps of Eucalyptus grandis and Eucalyptus urophylla using a pseudo-testcross: mapping strategy and RAPD markers. Genetics. 1994;137:1121-37.

40. Rounsaville TJ, Ranney TG. Ploidy levels and genome sizes of Berberis L. and Mahonia Nutt. Species, hybrids, and cultivars. HortScience. 2010;45:1029-33.

41. Chin C-S, Peluso P, Sedlazeck FJ, Nattestad M, Concepcion GT, Clum A, et al. Phased diploid genome assembly with single-molecule real-time sequencing. Nat Methods. 2016;13:1050.

42. Burton JN, Adey A, Patwardhan RP, Qiu R, Kitzman JO, Shendure J. Chromosome-scale scaffolding of de novo genome assemblies based on chromatin interactions. Nat Biotechnol. 2013;31:1119.

43. Haas BJ, Papanicolaou A, Yassour M, Grabherr M, Blood PD, Bowden J, et al. De novo transcript sequence reconstruction from RNA-seq using the trinity platform for reference generation and analysis. Nat Protoc. 2013;8:1494

44. Smith-Unna R, Boursnell C, Patro R, Hibberd JM, Kelly S. TransRate: reference-free quality assessment of de novo transcriptome assemblies. Genome Res. 2016;26:1134-44.

45. Simão FA, Waterhouse RM, loannidis P, Kriventseva EV, Zdobnov EM. BUSCO: assessing genome assembly and annotation completeness with single-copy orthologs. Bioinformatics. 2015;31:3210-2.

46. Koren S, Walenz BP, Berlin K, Miller JR, Bergman NH, Phillippy AM. Canu: scalable and accurate long-read assembly via adaptive k-mer weighting and repeat separation. Genome Res. 2017;27:722-36.

47. Chen $\mathrm{N}$. Using repeat masker to identify repetitive elements in genomic sequences. Curr Protoc Bioinformatics. 2004;5:4-10.

48. Srivastava R, Budak G, Dash S, Lachke SA, Janga SC. Transcriptome analysis of developing lens reveals abundance of novel transcripts and extensive splicing alterations. Sci Rep. 2017;7:11572.
49. Weirick T, Militello G, Müller R, John D, Dimmeler S, Uchida S. The identification and characterization of novel transcripts from RNA-seq data. Brief Bioinform. 2015;17:678-85.

50. Wang L, Cao C, Ma Q, Zeng Q, Wang H, Cheng Z, et al. RNA-seq analyses of multiple meristems of soybean: novel and alternative transcripts, evolutionary and functional implications. BMC Plant Biol. 2014;14:169.

51. Aghamirzaie D, Batra D, Heath LS, Schneider A, Grene R, Collakova E. Transcriptome-wide functional characterization reveals novel relationships among differentially expressed transcripts in developing soybean embryos. BMC Genomics. 2015;16:928.

52. He D, Liu Y, Cai M, Pan H, Zhang Q. The first genetic linkage map of crape myrtle (L. agerstroemia) based on amplification fragment length polymorphisms and simple sequence repeats markers. Plant Breed. 2014;133:138-44.

53. Myles S, Mahanil S, Harriman J, Gardner KM, Franklin JL, Reisch Bl, et al. Genetic mapping in grapevine using SNP microarray intensity values. Mol Breed. 2015:35:88.

54. Myburg AA, Griffin AR, Sederoff RR, Whetten RW. Comparative genetic linkage maps of Eucalyptus grandis, Eucalyptus globulus and their F1 hybrid based on a double pseudo-backcross mapping approach. Theor Appl Genet. 2003;107:1028-42.

55. Di Pierro EA, Gianfranceschi L, Di Guardo M, Koehorst-van Putten HJ, Kruisselbrink JW, Longhi S, et al. A high-density, multi-parental SNP genetic map on apple validates a new mapping approach for outcrossing species. Hortic Res. 2016;3:16057.

56. Chisholm ST, Coaker G, Day B, Staskawicz BJ. Host-microbe interactions: shaping the evolution of the plant immune response. Cell. 2006;124:803-14.

57. Jones JD, Dangl JL. The plant immune system. Nature. 2006:444:323-9.

58. Delaney TP, Uknes S, Vernooij B, Friedrich L, Weymann K, Negrotto D, et al. A central role of salicylic acid in plant disease resistance. Science. 1994;266:1247-50.

59. Lee H-A, Lee H-Y, Seo E, Lee J, Kim S-B, Oh S, et al. Current understandings of plant nonhost resistance. Mol Plant-Microbe Interact. 2017;30:5-15.

60. Seifi HS, Van Bockhaven J, Angenon G, Höfte M. Glutamate metabolism in plant disease and defense: friend or foe? Mol Plant-Microbe Interact. 2013:26:475-85.

61. Romero CC, Vermeulen JP, Vels A, Himmelbach A, Mascher M, Niks RE. Mapping resistance to powdery mildew in barley reveals a large-effect nonhost resistance QTL. Theor Appl Genet. 2018;131:1031-45.

62. Jafary $H$, Szabo LJ, Niks RE. Innate nonhost immunity in barley to different heterologous rust fungi is controlled by sets of resistance genes with different and overlapping specificities. Mol Plant-Microbe Interact. 2006;19:1270-9.

63. CADIC A. Breeding for ever-red barberries (Berberis spp.). Int Symp Sel Breed Woody Ornam. 1992. https://doi.org/10.17660/ActaHortic.1992.320.11.

64. Doyle JJ. A rapid DNA isolation procedure for small quantities of fresh leaf tissue. Phytochem Bull. 1987;19:11-5.

65. Poland JA, Brown PJ, Sorrells ME, Jannink J-L. Development of high-density genetic maps for barley and wheat using a novel two-enzyme genotypingby-sequencing approach. PLoS One. 2012. https://doi.org/10.1371/journal. pone.0032253.

66. Melo AT, Hale I. Expanded functionality, increased accuracy, and enhanced speed in the de novo genotyping-by-sequencing pipeline GBS-SNP-CROP. Bioinformatics. 2018. https://doi.org/10.1093/bioinformatics/bty873.

67. Margarido GRA, Souza AP, Garcia AAF. OneMap: software for genetic mapping in outcrossing species. Hereditas. 2007;144:78-9.

68. Melo AT, Guthrie RS, Hale I. GBS-Based Deconvolution of the Surviving North American Collection of Cold-Hardy Kiwifruit (Actinidia spp.) Germplasm. PLoS One. 2017. https://doi.org/10.1371/journal.pone.0170580.

69. van Os H, Stam P, Visser RG, van Eck HJ. SMOOTH: a statistical method for successful removal of genotyping errors from high-density genetic linkage data. Theor Appl Genet. 2005;112:187-94.

70. Kosambi DD. The estimation of map distances from recombination values Ann Hum Genet. 1943. https://doi.org/10.1111/j.1469-1809.1943.tb02321.x.

71. Voorrips RE. MapChart: software for the graphical presentation of linkage maps and QTLs. J Hered. 2002;93:77-8.

72. Broman KW, Wu H, Churchill G, Sen S, Yandell B. qtl: Tools for Analayzing QTL Experiments. R Package Version. 2010. https://rdrr.io/cran/qtl/

73. Haley CS, Knott SA. A simple regression method for mapping quantitative trait loci in line crosses using flanking markers. Heredity. 1992;69:315-24.

74. Stoffel $K$, van Leeuwen $H$, Kozik A, Caldwell D, Ashrafi $H$, Cui X, et al. Development and application of a 6.5 million feature Affymetrix Genechip ${ }^{\circledR}$ 
for massively parallel discovery of single position polymorphisms in lettuce (Lactuca spp.). BMC Genomics. 2012;13:185.

75. Chikhi R, Medvedev P. Informed and automated k-mer size selection for genome assembly. Bioinformatics. 2013;30:31-7.

76. Doležel J, Greilhuber J, Suda J. Estimation of nuclear DNA content in plants using flow cytometry. Nat Protoc. 2007;2:2233.

77. Roach MJ, Schmidt SA, Borneman AR. Purge Haplotigs: allelic contig reassignment for third-gen diploid genome assemblies. BMC Bioinform. 2018;19:460.

78. Quinlan AR, Hall IM. BEDTools: a flexible suite of utilities for comparing genomic features. Bioinformatics. 2010;26:841-2.

79. Camacho C, Coulouris G, Avagyan V, Ma N, Papadopoulos J, Bealer K, et al. BLAST+: architecture and applications. BMC Bioinform. 2009;10:421.

80. Gurevich A, Saveliev V, Vyahhi N, Tesler G. QUAST: quality assessment tool for genome assemblies. Bioinformatics. 2013:29:1072-5.

81. Quast C, Pruesse E, Yilmaz P, Gerken J, Schweer T, Yarza P, et al. The SILVA ribosomal RNA gene database project: improved data processing and webbased tools. Nucleic Acids Res. 2012;41:D590-6.

82. Chaisson MJ, Tesler G. Mapping single molecule sequencing reads using basic local alignment with successive refinement (BLASR): application and theory. BMC Bioinform. 2012. https://doi.org/10.1186/1471-2105-13-238.

83. Wu TD, Watanabe CK. GMAP: a genomic mapping and alignment program for mRNA and EST sequences. Bioinformatics. 2005;21:1859-75.

84. Li H, Durbin R. Fast and accurate short read alignment with BurrowsWheeler transform. bioinformatics. 2009;25:1754-60.

85. Dudchenko O, Shamim MS, Batra S, Durand NC, Musial NT, Mostofa R, et al. The Juicebox Assembly Tools module facilitates de novo assembly of mammalian genomes with chromosome-length scaffolds for under $\$ 1000$ bioRxiv. 2018. https://doi.org/10.1101/254797

86. Altschul SF, Madden TL, Schäffer AA, Zhang J, Zhang Z, Miller W, et al. Gapped BLAST and PSI-BLAST: a new generation of protein database search programs. Nucleic Acids Res. 1997;25:3389-402.

87. Li H. BFC: correcting Illumina sequencing errors. Bioinformatics. 2015;31:2885-7.

88. MacManes MD. Establishing evidenced-based best practice for the de novo assembly and evaluation of transcriptomes from non-model organisms. bioRxiv. 2016. https://doi.org/10.1101/035642

89. Bolger AM, Lohse M, Usadel B. Trimmomatic: a flexible trimmer for Illumina sequence data. Bioinformatics. 2014;30:2114-20.

90. Cantarel BL, Korf I, Robb SM, Parra G, Ross E, Moore B, et al. MAKER: an easyto-use annotation pipeline designed for emerging model organism genomes. Genome Res. 2008;18:188-96.

91. Bray NL, Pimentel H, Melsted P, Pachter L. Near-optimal probabilistic RNAseq quantification. Nat Biotechnol. 2016;34:525.

92. Pimentel H, Bray NL, Puente S, Melsted P, Pachter L. Differential analysis of RNA-seq incorporating quantification uncertainty. Nat Methods. 2017;14:687.

93. Goodstein DM, Shu S, Howson R, Neupane R, Hayes RD, Fazo J, et al. Phytozome: a comparative platform for green plant genomics. Nucleic Acids Res. 2011. https://doi.org/10.1093/nar/gkr944.

94. Consortium U. UniProt: the universal protein knowledgebase. Nucleic Acids Res. 2016. https://doi.org/10.1093/nar/gkw1099.

\section{Publisher's Note}

Springer Nature remains neutral with regard to jurisdictional claims in published maps and institutional affiliations.

Ready to submit your research? Choose BMC and benefit from:
- fast, convenient online submission
- thorough peer review by experienced researchers in your field
- rapid publication on acceptance
- support for research data, including large and complex data types
- gold Open Access which fosters wider collaboration and increased citations
- maximum visibility for your research: over 100M website views per year
At BMC, research is always in progress.
Learn more biomedcentral.com/submissions

